\title{
A Profile of Microbial Isolates and Antibiotic Susceptibility Patterns in Neonatal Sepsis at a Tertiary Care Centre in South India - A Post HOC Analysis
}

\author{
Santosh Kumar Kamalakannan ${ }^{1 *}$, NC Manikandan² and Sankavi Santosh Kumar ${ }^{3}$ \\ ${ }^{1}$ Department Of Neonatology, Saveetha Medical College and Hospital, India \\ ${ }^{2}$ Department Of Pediatrics, Madras Medical College and Research Institute, India
}

${ }^{3}$ Department Of Physical and Rehabilitation Medicine, Madras Medical College, India

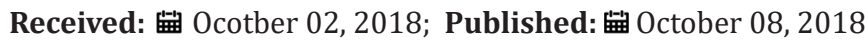

*Corresponding author: Santosh Kumar Kamalakannan, Department of Neonatology, Saveetha medical college and hospital, Thandalam, Chennai, India

\begin{abstract}
Background: Neonatal sepsis is a common problem in Neonatal intensive care units. Neonatal sepsis is classified as early onset and late onset based on the time of occurrence of clinical symptoms in the neonate. Organisms causing sepsis in neonates vary from unit to unit and from time to time within the same unit. Study of microbial pattern and their antibiotic sensitivity pattern is essential part of managing septic neonates.
\end{abstract}

Methods: The study was carried out in Govt Kilpauk medical college hospital between October 2010 to October 2012

Results: A total of 207 newborns with clinical sepsis were admitted. Blood culture was positive in 88 out of the 207 cases (42.51\%) Klebsiella is the commonest organism causing sepsis (45\%) in our Neonatal Intensive Care Unit. Other gram-negative isolates included Escherichia Coli (31\%), Pseudomonas (6\%), Enterobacter (1\%) and Acinetobacter (2\%). The Gram-positive organisms grown were Coagulase Positive and Coagulase negative Staphylococci (8\%), Group B Beta Hemolytic Streptococcus (5\%) and Enterococcus (2\%). Late-onset sepsis cases were found to be 1.5 times higher than early-onset sepsis. Out of 88 cases, $34(38.64 \%)$ had early-onset sepsis and 54(61.36\%) had late-onset sepsis.

Conclusion: A insight knowledge of the microbial isolate and their sensitivity pattern in Neonatal intensive care unit is essential in preventing mortality. The commonly pathogens isolated in the study are found to be resistant to the commonly used antibiotics.

Keywords: Neonatal Sepsis; Antibiotic Sensitivity; Gram Positive Organism; Multi Drug Resistant Organisms; Antibiotic Stewardship

\section{Introduction}

Neonatal sepsis is a clinical syndrome of bacteremia with systemic signs and symptoms of infection in the neonatal period $[1,2]$. Neonatal sepsis is broadly classified into two types namely early onset sepsis and late onset sepsis. Early onset sepsis is caused by organisms prevalent in the maternal genital tract, labour room or operating theatre, while late onset sepsis usually results from nosocomial or community-acquired infection. Sepsis is one of the major causes of mortality in neonates. According to World Health Organization (WHO) estimates, there are about 5 million neonatal deaths a year, with $98 \%$ occurring in developing countries [3]. The incidence of neonatal sepsis according to the data from National

Neonatal Perinatal Database (NNPD, 2002-03) is 30 per 1000 live births and contributes to $19 \%$ of all neonatal deaths [4]. Neonatal sepsis is caused by a variety of Gram +veas well as Gram -ve bacteria and rarely by fungus and yeast [5]. Diagnosis of neonatal sepsis clinically is difficult as the symptoms and signs are non-specific.

Hence a high index of suspicion is a must while managing a neonate with suspected sepsis. Antibiotics are the mainstay in the treatment of neonatal sepsis and must be stated at the earliest in case of late onset sepsis after obtaining the blood culture, while in case of early onset sepsis the decision to start antibiotics is governed by the presence of risk factors and the clinical signs and results of 
the Septic screen. Adjuvant therapy used in neonatal sepsis include GM-CSF, G-CSF, blood products and exchange transfusion on rare occasions. The organisms responsible for neonatal sepsis vary from place to place and with time even in a particular unit. Because of this changing pattern of organisms it is imperative to periodically audit the microbial pattern in the unit and change the management protocol periodically over time [6].

\section{Aim of the study}

This study was done to determine the microbial pattern and antibiotic sensitivity patterns of microbial isolates from blood cultures of neonates with sepsis, in Govt Kilpauk Medical College Hospital, a tertiary care center.

\section{Methodology}

An analysis was conducted on all blood culture reports obtained between 1st of October 2010 to the 1st of October 2012 from newborns admitted to the Department of Pediatrics and the Neonatal Intensive Care Unit (NICU) at Govt Kilpauk Medical College Hospital. Institutional Ethics committee approval and informed consent from the parents were obtained. The newborns were included in the study group if they satisfy any one of the following criteria.

Neonatal Criteria: Neonates showing the under-mentioned signs and symptoms, Respiratory distress or apnea or gasping respiration, Temperature instability - Hypothermia or Fever, Lethargy, poor cry, refusal of feeds, vomiting, ileus, abdominal distension, Poor peripheral perfusion, Bradycardia or Tachycardia, more than 10 pustules in the body or purulent umbilical discharge along with periumbilical erythema, Neonatal convulsions, irritability, hypotonia, altered sensorium.

Maternal Criteria: Maternal fever with evidence of bacterial infection within 2 weeks prior to delivery, Rupture of membranes more than 18 hours, Foul smelling liquor, Evidence of chorioamnionitis, More than 3 vaginal examinations or one unclean vaginal examination during labor, Prolonged labor (sum of first and second stage of labor more than 24 hours).

Blood Collection: Blood culture was done for all neonates suspected to have septicemia. Blood culture sample included a single sample collected from a peripheral vein under aseptic conditions. The local site was cleansed with $70 \%$ alcohol and povidone iodine (1\%), followed by $70 \%$ alcohol again. Blood cultures were incubated at $37^{\circ} \mathrm{C}$ and analysed at Microbiology department of Govt Kilpauk Medical College Hospital. Isolates were identified by the characteristic appearance on their respective media, Gram staining and confirmed by the pattern of biochemical reactions using the standard method and antibiotic sensitivity was also tested. C-reactive protein estimation and platelet count was also done as per unit policy before administration of empirical antibiotic

\section{Results}

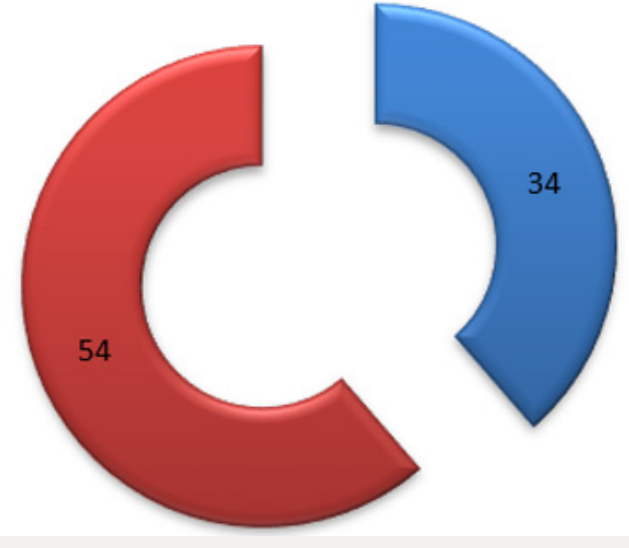

Early onset sepsis
- Late onset sepsis Mate onset sepsis

Figure 1: Blood culture positive.

During the study period, a total of 207 newborns with clinical sepsis were admitted. Of the 207 neonates admitted with suspected sepsis 112 were males and 97 were female. Male:female ratio was 54.1\%:45.9\%. On categorizing the newborns based on birth weight, 11 babies were of very low birth weight, 107 newborns were of low birth weight, 88 babies were in normal birth weight and one baby weighed more than 4,000 grams. Blood culture was positive in 88 out of the 207 cases (42.51\%). Klebsiella is the commonest organism causing sepsis (45\%) in our Neonatal Intensive Care
Unit. Other gram-negative isolates included Escherichia Coli (31\%), Pseudomonas (6\%), Enterobacter (1\%) and Acinetobacter (2\%). The Gram-positive organisms grown were Coagulase Positive and Coagulase negative Staphylococci (8\%), Group B Beta Hemolytic Streptococcus (5\%) and Enterococcus (2\%). Late-onset sepsis cases were found to be 1.5 times higher than early-onset sepsis. Out of 88 cases, 34 (38.64\%) had early-onset sepsis and 54(61.36\%) had late-onset sepsis. Best overall sensitivity among Gram-negative isolates was to Imipinem (92\%) followed by Amikacin (82.66\%) and 
best sensitivity among Gram-positive isolates was to Vancomycin 88 positive cultures 6 were multi-drug resistant (6.8\%) (Figures (84.61\%), followed by Cloxacillin and Ampicillin (61.53\%). Out of 1-5).

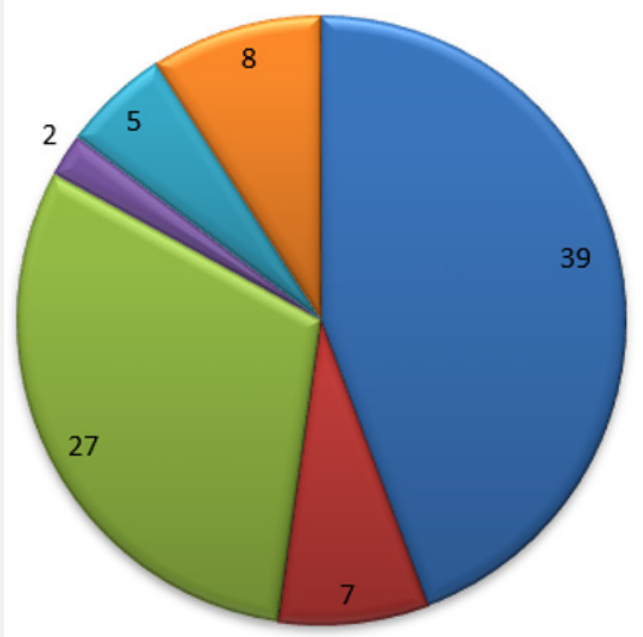

曰 KLEBSIELLA

ISTAPH. AUREUS

$\square$ E. COLI

DENTEROCOCCI

$\square$ PSEUDOMONAS

Figure 2: Common causative organisms (Total=88).

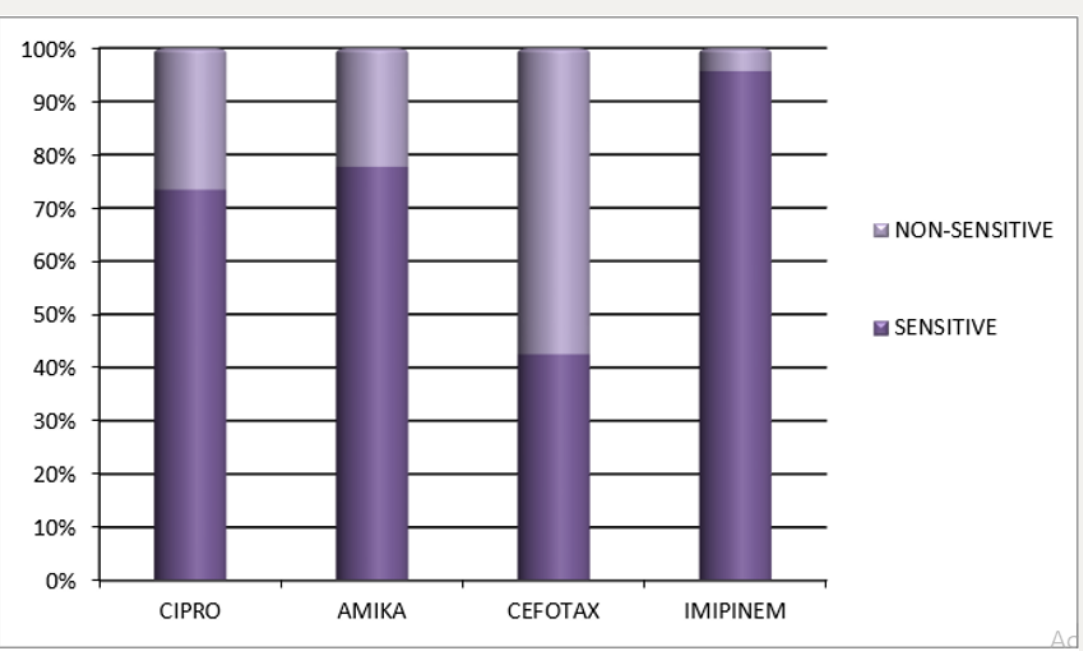

Figure 3: Antibiotic sensitivity pattern of Klebsiella (Total=39).

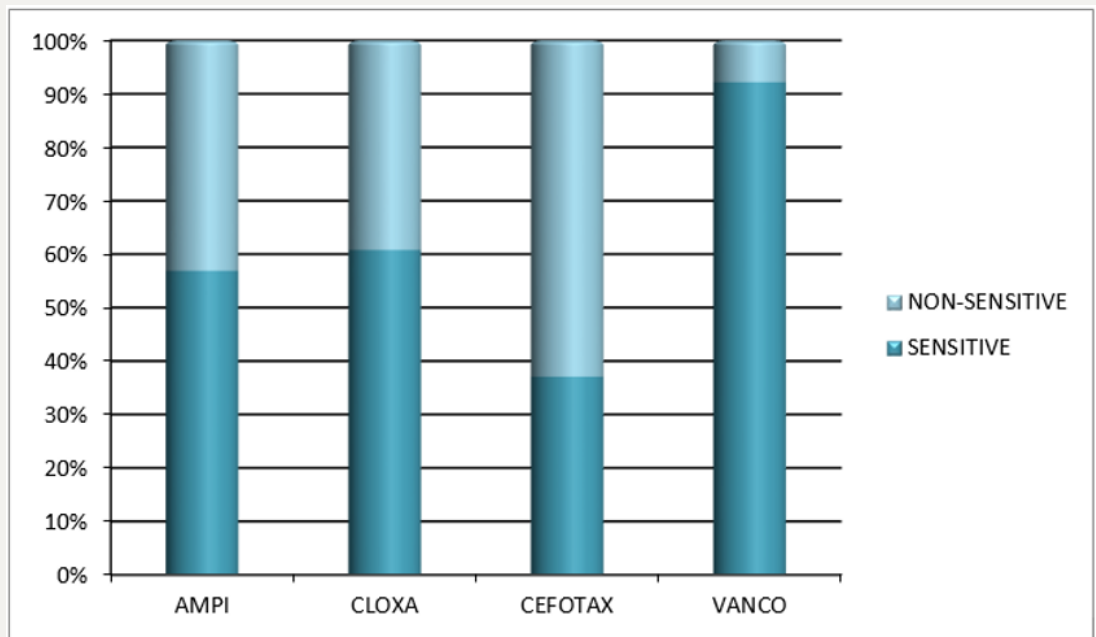

Figure 4: Antibiotic sensitivity pattern of Staphylococcus Aureus (Total=7). 


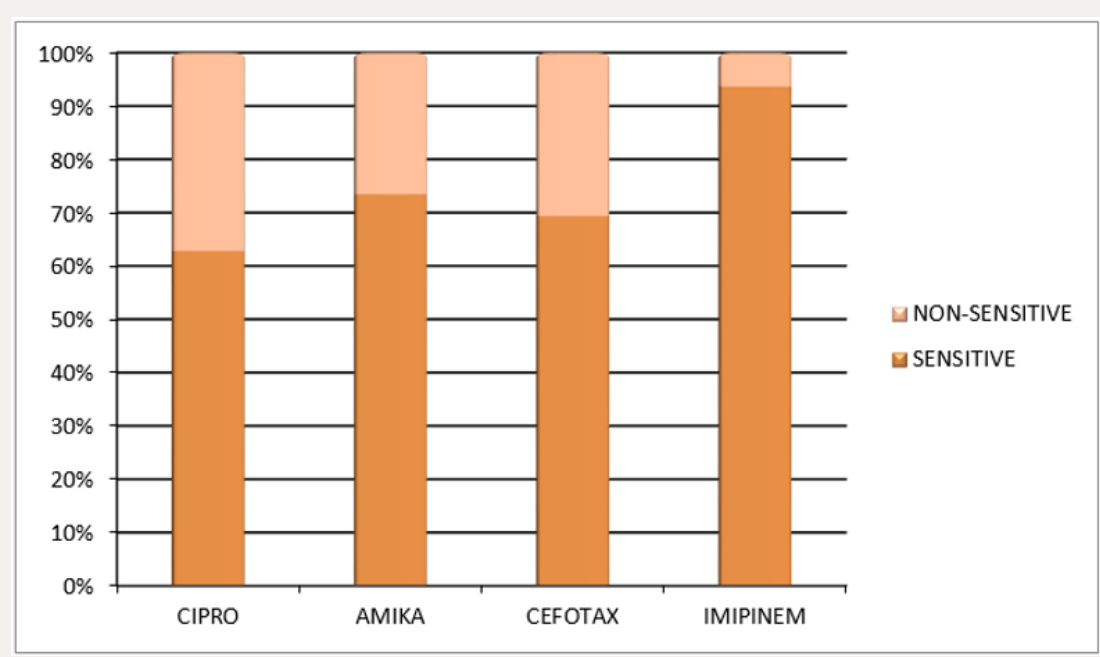

Figure 5: Antibiotic sensitivity pattern of other organisms (E.Coli, Streptococci, Pseudomonas, Acinetobacter) (Total=42).

\section{Discussion}

The organisms implicated in neonatal sepsis in developing countries differ from those seen in developed countries [3]. In our study, out of 88 positive cultures, majority of causative organisms were Gram negative (85.22\%), and specifically, the most common organisms were Klebsiella (45\%) and E.coli (31\%). Among the Grampositive organisms Staphylococcus aureus (8\%) predominated. It is consistent with the data from NNPD 2000, that the most common organisms responsible for neonatal sepsis in India are Klebsiella [1]. Gram-negative and Gram-positive septicemia was encountered in $85.22 \%$ and $14.78 \%$ of the culture-positive cases in our study which is in contradiction to results of study by Agnihotri et al. [7] where a predominace of gram negative organism was encountered but gram positive isolates contributed to nearly $42 \%$ of sepsis cases .This could be possibly explained by the fact that most of our cases were late onset sepsis cases and even among early onset sepsis cases there was a predominance of gram negative organisms.

The analysis of drug resistance pattern showed that, among Gram-negative isolates nearly $60 \%$ were resistant to cefotaxime and $66 \%$ resistant to ciprofloxacin which are commonly used a first line drugs. Six out of 88 isolates were multidrug resistant and this was more common among the common pathogen isolated (Klebsiella and E.Coli). Prevalence rate of Group B streptococci is comparable to the results of Donal Waters et al. [8].

\section{Conclusion}

There cannot be a single recommendation for the antibiotic regimen for neonatal sepsis in all settings. The choice of antibiotics depends on the prevailing flora responsible for sepsis in the given unit and their antibiotic sensitivity. Microbial Isolates seem to be resistant to commonly used antibiotic for neonatal sepsis, cefotaxime and ciprofloxacin. There is an increase in the multidrug resistant (ESBL) Gram Negative which needs vigilant surveillance.

\section{References}

1. http://www.newbornwhocc.org/pdf/sepsis.pdf

2. emedicine.medscape.com

3. Vergnano S, Sharland M, Kazembe P, Mwansambo C, Heath PT (2005) Neonatal sepsis: An international perspective. Arch Dis Child Fetal Neonatal Ed 90(3): F220-F224.

4. Shalini Tripathi (2010) Neonatal sepsis: past, present and future. Internet Journal of Medical Update 5(2): 45-54.

5. Huda HA Gomaaa, Edet E Udo, Usha Rajaram (2001) Neonatal septicemia in AlJahra hospital, Kuwait: Etiologic agents and antibiotic sensitivity patterns. Med Princ Pract 10:145-150.

6. P Shrestha, BK Das, NK Bhatta, DK Jha, B Das, et al. (2007) Clinical and bacteriological profiles of blood culture positive sepsis in newborns. J Nepal Paediatr Soc; 27(2): 64-67.

7. Agnihotri N, Kaistha N, Gupta V (2004) Antimicrobial susceptibility of isolates from neonatal septicemia. JpnJ Infect Dis 57(6): 273-275.

8. Waters D, Jawad I, Ahmad A, Lukšić I, Nair H, et al. (2011) Aetiology of community-acquired neonatal sepsis in low and middle income countries. J Glob Health 1(2): 154-170. 
(C) (- This work is licensed under Creative

To Submit Your Article Click Here: Submit Article

DOI: $10.32474 /$ PAPN.2018.01.000123

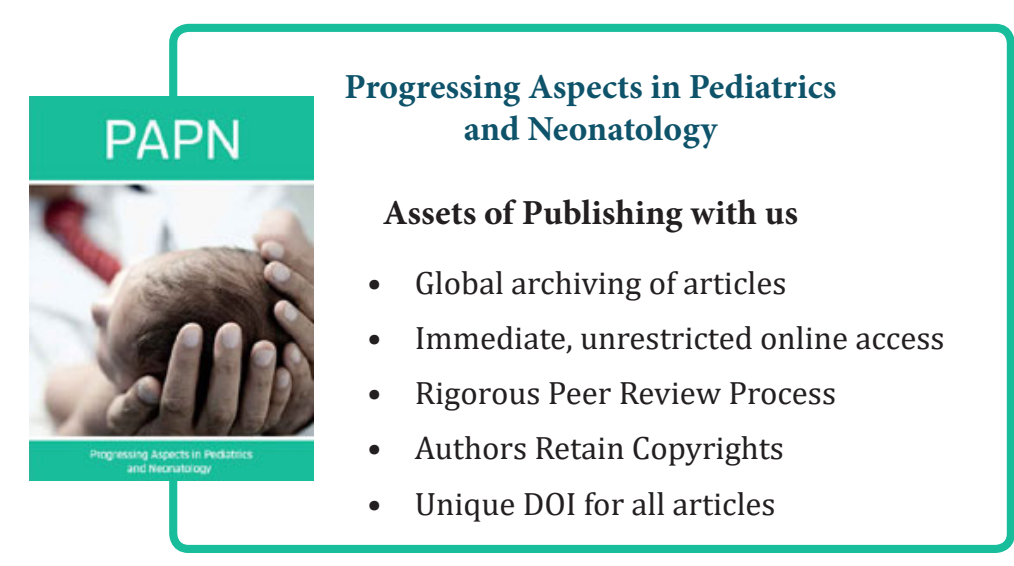

